\title{
Geometrical Modeling of Crystal Structures with Use of Space of Elliptic Riemannian Geometry
}

\author{
Stanislav Rudnev ${ }^{1}$, Boris Semukhin ${ }^{2}$, Andrey Klishin ${ }^{1}$ \\ ${ }^{1}$ Tomsk Polytechnic University, Tomsk, Russia; ${ }^{2}$ Institute of Strength Physics and Materials Science SB RAS, Tomsk, Russia. \\ Email: stas_rudnev2004@mail.ru, bss@ispms.tsc.ru, aklishin@yandex.ru \\ Received February $28^{\text {th }}, 2011$; revised March 21 $1^{\text {st }}, 2011$; accepted April $6^{\text {th }}, 2011$.

\begin{abstract}
The space of internal geometry of a model of a real crystal is supposed to be finite, closed, and with a constant Gaussian curvature equal to unity, permitting the realization of lattice systems in accordance with Fedorov groups of transformations. For visualizing computations, the interpretation of geometrical objects on a Clifford surface $\left(S_{K}\right)$ in Riemannian geometry with the help of a $2 D$ torus in a Euclidean space is used. The F-algorithm ensures a computation of $2 D$ sections of models of point systems arranged perpendicularly to the symmetry axes $l_{3}, l_{4}$, and $l_{6}$. The results of modeling can be used for calculations of geometrical sizes of crystal structures, nanostructures, parameters of the cluster organization of oxides, as well as for the development of practical applications connected with improving the structural characteristics of crystalline materials.
\end{abstract}

Keywords: F-Algorithm, Crystal Lattice Systems, Microstructure, Riemannian Geometry, Space of Interpretation

\section{Introduction}

Modern material science, building on very different models of the structure of a substance, tries to create absolutely new materials or materials with properties needed when exploiting machines and mechanisms under unusual conditions. Let us note that some successes are recently observed in making materials with a structure modeled at very different scaled and dimensional levelsit is the so-called nanomaterials technology. However, essential breakthroughs in applications of these materials when fabricating microelectronic engineering have not yet been made. In our opinion, an essential value has the fact that all up-to-date models are based only on one notion of the structure of a solid, namely, considered in a Euclidean space.

In the present paper we offer the other, alternative approach to describing both a structure and making materials with special or unique properties by means of interpreting the space of experience and processing materials in a strictly symmetrized electromagnetic field.

Development of physicochemical methods of investigating a crystal structure and processing technologies, growing needs for production of high quality crystalline materials arouse a lot of attention to new approaches grounded on modeling crystal structures with the use of different modeling spaces. At the present time, concepts of non-Euclidean phase spaces are ever more widely used for describing general evolutionary principles of various physical systems. In this connection, a particular interest of researchers to problems of a possible realization of Fedorov groups in non-Euclidean spaces is noted. The realization of Fedorov groups of symmetry was considered in a pseudo-Euclidean space, Lobachevsky space and Minkowski space [1]. A lack of similar examinations is that in the specified spaces a space of infinite extent is used for a model of lattices $[2,3]$.

It is traditionally accepted to construct the crystal structure of an ideal crystal by the multiplication of a finite number of atoms by all transformations from some Fedorov group in a Euclidean space. But such a construction of an ideal crystal is not related to natural causes of the growth of a real crystal, its finite (localised) character and shape restricted in the space. Besides, the problem of the relation of the internal structure and external faceting of a real crystal remains unresolved. An ambiguity of the geometrical interpretation of the space of the real crystal of minerals has led to the necessity of developing models by using a non-Euclidean method of describing crystal structures.

When modeling under the conditions of Riemannian geometry, it will be natural to maintain the term "Fedorov group" for discrete groups of movements, which 
we shall denote by $F$-groups. For a model of the crystal structure in an elliptic space, as well as in the Euclidean case, the conditions of the global discreteness and homogeneity are fulfilled. In the case of $F$-groups the finiteness theorem for the volume of the fundamental domain of a finite polyhedron is rather simply proved.

When modeling crystal structures, the space of Riemannian geometry $\left(V_{4}\right)$ with the constant Gaussian curvature $K=1$, that coincides in sufficiently small regions with a Euclidean space (a locally Euclidean space), was chosen as a modeling space. When constructing crystal lattices, simulated groups of transformations, which are used for computations of point systems, are the basic tool of investigating. In this case we deal with a finite space where the distance between any two points does not exceed a certain value [4-8].

\section{Geometrical Approach to Modeling Crystal Structures}

\subsection{Space of Interpretation $\boldsymbol{R}_{E}$}

Historically developed priority of Euclidean geometry (despite the discoveries of non-Euclidean geometries) has led to two fundamental consequences:

1) Up till now, the space of our experience is supposed to be Euclidean,

2) All the laws of physics and chemistry are supposed to be realizing in a Euclidean space.

And though mathematicians and, partly, physicists do not forget to repeat that with N. Lobachevsky, J. Bolyai and B. Riemann's discoveries Euclidean geometry has lost its unique position as the singular geometry of the space of our experience, the problem of the choice of an adequate geometrical space is still open. Nevertheless, a reliable experimental confirmation of the fulfillment of Coulomb's law in non-Euclidean spaces has not yet been obtained, and so far it is not clear how it can be realized.

However, after A. Poincaré constructed the interpretation (a model) of a non-Euclidean geometry (realizing Lobachevsky's plane), the situation changed radically. It turned out that Euclidean geometry itself and, accordingly, a Euclidean space are no more than one of a variety of geometrical interpretations. There arises the unique possibility of considering the interpretation (representation) of one geometry by means of geometrical images of other geometry, of course, according to strictly defined rules.

A considerable quantity of modern investigations which can be found in [1] is devoted to interpretation problems. For constructing geometrical models of crystal structures the approach applied in [9] has been used.

A general scheme for the interpretation of elliptic Riemannian geometry in a Euclidean space $\left(I_{E}^{R}\right)$ is simple enough.

A certain geometrical image $A$ of the Riemannian space $A \in V_{4}$, possessing necessary properties, is chosen as an object of the interpretation in a Euclidean space, with the help of some geometrical image $B$ of the Euclidean space, $I_{E}^{R}(A)=B$, where $B \in R^{3}$. The chosen geometrical image $B$ of the Euclidean space is endowed with properties of the geometrical image $A$, and becomes, thus, a carrier of properties of the geometrical image of the Riemannian space in the Euclidean space.

Operations with the geometrical image $B$ are executed according to the rules of the Riemannian space with regard to certain conditions stated below. Consequently, in the Euclidean space there arises a certain region $R_{E}$ which is called the space of interpretation.

$$
I_{E}^{R}(M)=R_{E}
$$

where $M$ is the Riemannian space being subject to the interpretation.

Properties of the space $R_{E}$ are sufficiently specific and taken into consideration in each specific case.

A Clifford surface $S_{K}$-a direct circular cylinder of the elliptic space [10] is chosen as the basic geometrical element of the elliptic Riemannian space for constructing the space of interpretation $R_{E}$. The basis for such a choice is the fact that Euclidean geometry $\left(R^{2}\right)$ takes place on $S_{K}$.

D. Hilbert wrote about it that the greatness of W. Clifford's discovery is that the Euclidean plane "in the small" is present in a closed and restricted curvilinear space. $S_{K}$ is isomeric to a Euclidean rectangle or rhomb with identified opposite sides, what leads us to an ordinary Euclidean torus.

$$
I_{E}^{R}\left(S_{K}\right)=T_{2}
$$

where $T_{2}$ is a $2 \mathrm{D}$ torus belonging to the Euclidean space.

But with that principal difference, that a torus loses its geometrical independence in the space of interpretation and becomes a carrier of the $S_{K}$ properties. That is, in the space of interpretation a torus is a very surface on which Euclidean geometry is fulfilled and well-known groups of movements of a Euclidean torus are more not applicable to it. To operate with a torus in the space of interpretation one should follow other rules. In $R_{E}$ the motions of a torus are rotations. At the same time, using the fact that $R_{E}$ is "arranged" in a Euclidean space, it is possible to carry out sections of $R_{E}$ by a Euclidean plane and, in such a way, to study properties of structures in the space of interpretation. It is extremely important that the Clifford surface is a carrier of Euclidean geometry, then in $R_{E}$ on a torus (or on systems of tori) all the physical laws show themselves in the same form and with the same content, 
as on the Euclidean plane and in the space. The difference is that an arc lying on a torus is used instead of a segment of a Euclidean straight line. For example, in Coulomb's law $L$-an arc on a torus is used in $R_{E}$ instead of $r$.

The existence of systems of parallel straight lines of the Riemannian space on the Clifford surface $\left(S_{K}\right)$ leads to the presence of groups of parallel transports, both on the Clifford surface and generally in the Riemannian space (the so-called Clifford's parallels), and, so, of groups of translations. It is important to remember that these groups are realized only on $S_{K}$, but not on planes of the Riemannian space. In $R_{E}$ these groups show themselves rather originally and will be described below. Images of translations on the Clifford surface are expressed in $R_{E}$ as a rotation of the torus about own axes, and are defined by the group $H_{T}=\left\{H_{T}^{l_{1}}, H_{T}^{l_{2}}\right\}$, where $H_{T}^{l_{1}}$ is a subgroup of the rotation about the axis $l_{1}, H_{T}^{l_{2}}$ is a subgroup of the rotation about the axis $l_{2}$, that is, as a slip on itself.

$$
I_{E}^{R}\left(H_{S}\right)=H_{T},
$$

where $H_{S}$ is a translational group formed from subgroups of the paratactic displacements of $S_{K}$.

Translations of the Clifford surface itself in the Riemannian space are expressed in $R_{E}$ as a rotation of the torus about own generatrices.

$$
I_{E}^{R}\left(H_{S}\left(S_{K}\right)\right)=H_{T}\left(T_{2}\right) .
$$

Different crystallographic axes of symmetry (from $l_{2}$ up to $l_{6}$ ) are considered on different Clifford surfaces, what is expressed in $R_{E}$ as an application of tori with different ratios of the interior and exterior radiuses. When unfolding tori, it shows itself in the form of rectangles with different lengths of sides and with different angles between their diagonals (for the axis $l_{3}$, for example, an angle between diagonals is taken to be equal to 60 or 30 degrees, for the axis $l_{4}$ this angle is equal to 45 degrees, with corresponding side lengths of the rectangle of an unfolding).

$$
I_{E}^{R}\left(H_{T}^{m_{i}}\left(S_{K}\right)\right)=H_{T}^{l_{i}}\left(T_{2}\left(r_{i}, R_{i}\right)\right),
$$

where $r_{i}$ and $R_{i}$ are the interior and exterior addresses of a torus, respectively; $H_{T}^{l_{i}}$ are subgroups of the rotation of $T_{2} ; H_{S}^{m_{i}}$ are subgroups of the rotation of $S_{K} ; i$ is an order of symmetry.

$$
I_{E}^{R}\left(O_{S}\right)=O_{T},
$$

where $O_{T}=\left\{H_{T}\left(T_{2}^{O}\left(r_{k}, R_{k}\right)\right)\right\}$ is a subgroup of the rotation of a torus, the ratio $\frac{r_{k}}{R_{k}}=\frac{m}{n}$ being correct for the radiuses.
By the space of interpretation $R_{E}$ (Riemannian in Euclidean) we shall call a totality of all points $A$ being equivalent to a point $O$ through which there passes a torus- $T_{2}^{O}\left(r_{k}, R_{k}\right)$ with the ratio of the internal and exterior radiuses being equal to $m: n$, on all possible translations of this torus, interpreted as it was pointed out above.

$$
R_{E}=\left\{H_{T}, O_{T}\right\},
$$

where $H_{T}$ is a translational subgroup; $O_{T}$ is a subgroup of the rotations that form a subspace of $R_{E}$. We shall call the point $O$ by the center of the space of interpretation. It is easy to show that the space of interpretation is restricted, closed, and continuous. A select of the value of the ratio of the radiuses of a torus will be defined for a given model by a type of symmetry of a crystal structure viewed. It is convenient to study the geometrical and structural features of objects in $R_{E}$ by means of sections of the space of interpretation by Euclidean planes. However, the most important property of the space of interpretation is that on a torus in $R_{E}$ we have the right to consider physical laws in their normal Euclidean interpretation, except for a replacement of Euclidean segments by arcs of a torus.

For problems of mineralogy, crystallography and material science Coulomb's law is of heightened interest - thanks to its simplicity and the fact that when interpreting it the features of $R_{E}$ show themselves most brightly, namely:

- In the space of interpretation there exist three families of the shortest lines (rounds with the ratio of diameters being equal 1:2:3) each of which becomes a family of lines of force of an electrostatic charge $Q$. The electrostatic lines of force do not start from the charge $Q$, and do not end at infinity, but touch a surface of the charge, and close up at some distance from it. This distance is calculated using usual trigonometry formulas;

- An electrostatic field of a charge is restricted in sizes, closed, and continuous. At some distance from a charge $Q$ its electrostatic field is already absent. This distance is called a radius of the field of a charge $Q$ and denoted by $R_{p}$. It should be noted that an electrostatic field of a charge $Q$ does not occupy the entire space of interpretation completely. Thus two different charges, $Q_{1}$ and $Q_{2}$, can interact or not, depending on a distance between them, as well as on their own linear dimensions.

Studying the features of crystal structures in $R_{E}$ is possible in sections by its Euclidean plane in two ways:

Case 1: Symmetry of a structure is known. A model of a crystal structure is considered in the form of point systems where points simulate a distribution of centers of 
atoms in lattice points - the so-called $R$-systems which are computer-simulated using a special program. A section by the Euclidean plane is carried out either perpendicularly to the axis of symmetry of the structure studied, or at an angle interesting us.

Case 2: Symmetry of a structure is unknown, or a structure has been badly studied from the point of view of symmetry. Under these circumstances the task becomes complicated and is solved in a few stages, what will be considered below (Item 1.2).

The principal difference of an ideal lattice system in a Euclidean space and in $R_{E}$ consists in the following: in a Euclidean space an ideal crystal has to be either infinite large-sized or infinitesimal. An ideal Euclidean crystal has no center of symmetry, zonality, and sectoriality, and is not faceted. The question about the faceting of an ideal crystal is solved with the help of introducing different sets of the so-called "boundary conditions". Simply speaking, the ideal Euclidean model of a crystal does not possess those structural features that are characteristic for real crystals, except for a fragmentary coincidence to restricted fragments of a plane lattice. In the history of mineralogy, crystallography, and geology generally, no real crystal coinciding on its own structural and symmetry characteristics with its ideal Euclidean models has been met. The fact is well-known, but somehow slips attention of researchers all the time.

In the space of interpretation $R_{E}$ there is the other situation. An ideal crystal in the space of interpretation is restricted in sizes, has a certain shape and symmetry, is zonal and sectorial, and also possesses the center - that is, it possesses practically a complete set of the structural and symmetry characteristics which the real crystal of a mineral has. If one takes into account that both the radius of an electrostatic field $R_{p}$ and linear dimensions of the space of interpretation $R_{E}$ are calculated in accordance with values of the ionic radius $R_{i}$ and atomic radius $R_{a}$ of a given substance, we can always estimate both real sizes of microcrystalline blocks and distances at which there are electrostatic interactions between real ions.

\subsection{Principles of Modeling Crystal Structures in Elliptic Riemannian Geometry}

In modern structural examinations, when describing the organization and processes of the growth of a crystal structure, information about the type of a space in which an explored process being watched is not enough used [11-14]. The examinations of different non-Euclidean methods of describing elements of a crystal lattice [4] are the foundation of the suggested theoretical approach to modeling crystal structures under the conditions of Riemannian geometry. In order to achieve results the interpretation of the geometrical objects $\left(S_{K}, F\right.$-groups, and symmetries) in Riemannian geometry is used. In a three-dimensional Euclidean space a $2 \mathrm{D}$ torus $T_{2}$ (see formula 2), on which the basic geometrical transformations (lattices, elements of Fedorov groups) are considered, corresponds to the Clifford surface $\left(S_{K}\right)$.

The basic difference from existing approaches to modeling consists in a statement according to which the organization of a lattice structure happens in accordance with a certain $F$-group $\left(I_{E}^{R}(F)=\Phi\right)$ operating in the Riemannian space $V_{4}$. An atom or ion in a model of the lattice system is considered as a point site, and point systems are studied in the initial stage without regard to the chemical features of atoms. By using the approach under consideration, modeling the cluster organization of microstructures of oxides is carried out, as well as practical applications aimed at perfecting crystalline materials and improving their physical properties are developed.

General principles of modeling crystal structures in elliptic Riemannian geometry may be reduced to the following:

- Theoretical modeling of lattice structures of a given crystal in accordance with its Fedorov group of symmetry, realized in the elliptic Riemannian space with the involvement of visualizing the model constructions. Building on data obtained when modeling point systems with a given Fedorov group, it is possible to form judgments about the morphology of a structure, clustering, and a type of zonality, and to make suppositions of different types of anisotropy;

- Theoretical modeling of a family of structures of electrostatic fields of ions of elements having participated in the formation of lattices of a given crystal; constructing the potential surfaces answering to minimum energy;

- Theoretical modeling of microstructures of crystals in the model sections perpendicular to axes of symmetry, with regard to: a composition, symmetry, a structure of electrostatic fields of ions and their systems, the principle of closest packing; as well as crystallographic analysis and interpreting data obtained.

Thus a general procedure of computing experiment for obtaining a model of a crystal structure of a necessary chemical composition and structure adds up to the following stages.

Stage 1: The chemical composition of an explored structure is detected.

Stage 2: The structure of electrostatic components of parameters of ions and atoms constituting the structure of a given substance is computed.

Stage 3: Regularities of constructing sublattices for each of elements composing a given structure are revealed, according to the series of crystallochemical activity [15]. 
Stage 4: Upon the Clifford surface's unfolding a fragment of the sublattice is constructed using the computations of the parameters of electrostatic components of a model for each ion, and then the incorporation of sublattices of the rest of atoms and ions is conducted in accordance with the computed parameters, according to the series of crystallochemical activity [15].

Stage 5: By the unfolding obtained an appropriate torus is folded, and all the necessary transformations (parallel translations, rotations about axes of symmetry, etc.) are applied to it, according to the scheme for the interpretation $I_{E}^{R}$.

Stage 6: For visualizing the constructions a necessary section of the space $R_{E}$ by a Euclidean plane is constructed, where the features of a studied structure are considered.

\section{3. $F$-Algorithm}

The important place in the examination of properties and features of the organization of a crystal structure in the space $R_{E}$ is occupied with constructions of point systems with the use of special computational algorithms for solving of tasks of the visualization and interpretation of constructed models. For modeling lattice systems in the space $R_{E}$ an algorithm has been developed, with the help of which constructions of lattices are produced with regard to the fact that for each Fedorov group $\Phi$ of the Euclidean space $R^{3}$ in the Riemannian space there is an appropriate $F$-group satisfying to the condition $I_{E}^{R}(F)=\Phi$.

For the proof it is enough to take the projective space $R P^{3}$ and to use the homeomorphism theorem for the groups $S O(3)$ and $S U(2)$ [16]. From an epimorphism $\pi: S U(2) \rightarrow S O(3)$ it is possible to obtain $F$-groups, as subgroups of the group $S U(2)$. The so-called binary groups arise:

$$
\begin{aligned}
& D_{n}^{*}=\pi^{-1}\left(D_{n}\right), \quad T_{n}^{*}=\pi^{-1}\left(T_{n}\right), \quad Q_{n}^{*}=\pi^{-1}\left(Q_{n}\right), \text { and } \\
& I_{n}^{*}=\pi^{-1}\left(I_{n}\right),
\end{aligned}
$$

a binary group of a dihedral, a binary group of a tetrahedron, a binary group of an octahedron, and a binary group of an icosahedron. The binary groups, as well as orthogonal representations, in whole, arise naturally in describing a physical system with a spin and in calculating characteristics of gravitational fields in Riemannian spaces.

The algorithm suggested in this work defines rules for constructing point systems for a given $F$-group and therefore will be denoted by $F$-algorithm. The given $F$-algorithm, realizing the translational subgroup $H_{S}$ in the Riemannian space when interpreting $I_{E}^{R}\left(H_{S}\left(S_{K}\right)\right)=$ $H_{T}\left(T_{2}\right)$, will describe a representation by the following rule for a point on a Euclidean plane:

$$
\begin{gathered}
r_{1}=\sum_{s=1}^{3} \exp \left(\frac{2 \pi l_{s} i}{n}\right), l_{s} \in(1, n), \\
r=\boldsymbol{G}_{F} r_{1} .
\end{gathered}
$$

As parameters of a model the following variables were accepted: $\boldsymbol{G}_{F}$ is a matrix of a subgroup of the rotations $O_{T}$ induced in a section by a given $F$-group, where $I_{E}^{R}(F)=\Phi ; r_{s}$ is the radius of the surface $S_{K} ; r_{t}$ is the radius of an appropriate torus $T_{2}$, where $I_{E}^{R}\left(r_{s}\right)=r_{t} ; n$ is an amount of the points lying on the section of the surface $S_{K}$.

A set of points computed by the $F$-algorithm (8) organizes a system of the points on a plane, which we shall denote by $K$-system. The parameters defining a $K$-system are: a Fedorov group $\Phi, F$-groups, and a basic axis of symmetry $l_{i}$. A $K$-system can be determined with the help of its constituent sets $T_{n}^{i}$ and represented in the following form:

$$
K=\left\{T_{n}^{1}, T_{n}^{2}, T_{n}^{3}\right\},
$$

where $T_{n}^{i}$ are sequentially generated sets in Figure 1 (for the case $n=6$ ), satisfying to the conditions given below:

$$
\begin{aligned}
& T_{n}^{1}=\left\{\left(x_{i}, y_{i}\right)_{O} \mid x_{i}^{2}+y_{i}^{2}=r_{t}^{2}, i=1, n\right\}, \\
& T_{n}^{2}=\left\{\left(x_{j}, y_{j}\right)_{(x, y)} \mid x_{j}^{2}+y_{j}^{2}=r_{t}^{2},(x, y) \in T_{n}^{1}, i=1, n\right\}, \\
& T_{n}^{3}=\left\{\left(x_{k}, y_{k}\right)_{(x, y)} \mid x_{k}^{2}+y_{k}^{2}=r_{t}^{2},(x, y) \in T_{n}^{2}, k=1, n\right\} .
\end{aligned}
$$

A set of points computed by the $F$-algorithm (9) organizes a system of the points on a plane, which we shall denote by $R$-system. This implies that the generation of an $R$-system is carried out by performing a group of the transformations $H_{T}\left(T_{2}\right)$ over a $K$-system.

$$
R=\left\{H_{T}\left(T_{2}\right) K\right\} .
$$

The initial forms of the organization of real crystals are $R$-systems. The real structure of a crystal is supposed to be consisting of rows of particles, integrated into a system, and to have concrete sizes of the space of interpretation. With the help of the apparatus of elliptic Riemannian geometry, a distribution of atoms and a filling of the crystalline space can be interpreted as a compact locally Euclidean set. The misorientation and displacement of $R$-systems are accompanied by the appearance, in the integrated space of a crystal, of blocks and boundary spaces separating them [15].

The process of the organization of a crystal structure in $R_{E}$ consists in sequential introducing new particles into consideration, arranged at the shortest distances (put on a torus) from fixed ones in accordance with a given Fedorov 


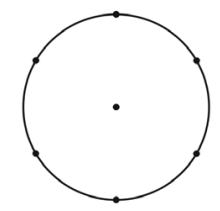

(a)

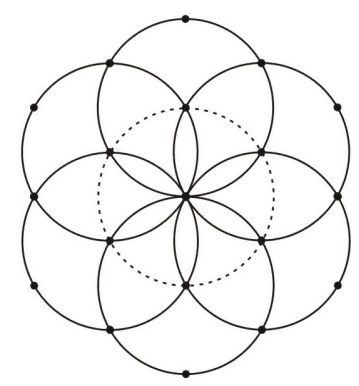

(b)

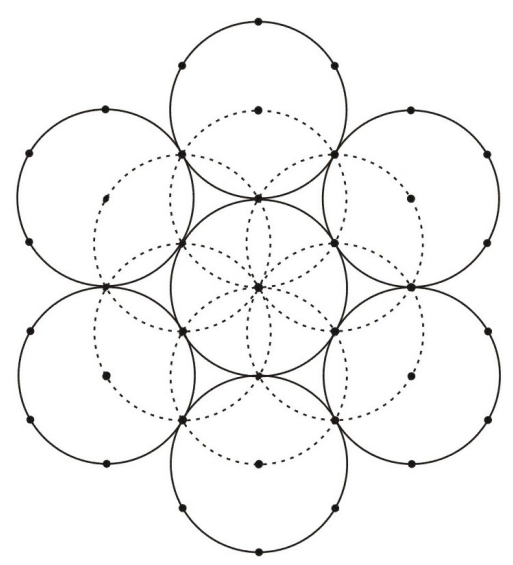

(c)

Figure 1. Stages of constructing a $K$-system. A sequence of the formation of the sets: (a) $T_{6}^{1}$; (b) $T_{6}^{2} ;$ (c) $T_{6}^{3}$.

group, up to the faceting. Thus the organization of a lattice in $R_{E}$ can be presented as a multistage process in which bonding of chemically responsive particles is under way, beginning from the zero point.

On a $K$-system, nodes are connected by arcs of circles and arranged from each other at a certain number of the lattice periods $(E)$. An ideal crystal is usually constructed by the multiplication of a finite number of atoms by all the transformations of some Fedorov group, but such a construction of the ideal crystal is not related to natural causes of the crystallization [4].

The organization of a lattice system in the space of interpretation $R_{E}$, up to the faceting of a crystalline polyhedron, consists of the structurally-forming (Figure 2(a)) and structurally-filling (Figure 2(b)) stages, each of newly fixed particles behaving like a new center of the organization of the lattice. The realization of bonds passes along geodetic lines of the elliptic space, spreading the

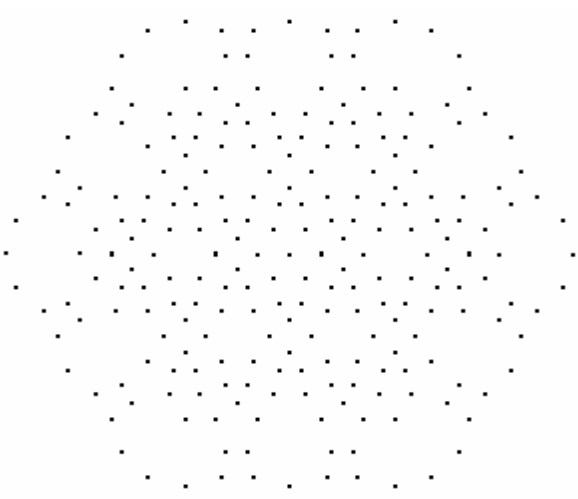

(a)

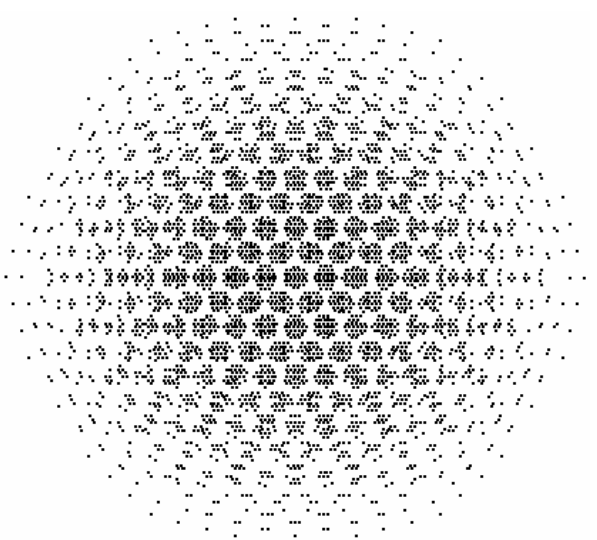

(b)

Figure 2. (a) Structurally-forming ( $T_{12}^{1}$ ), and structurally-filling stages of the organization of a crystal structure; (b) The sets $\left(T_{12}^{2}, T_{12}^{3}\right)$ have been constructed.

forces of long-range interaction to the whole closed region, what is a specific feature of an elliptic Riemannian space.

The noted features of a distribution of lattice points on a $R$-system are in good conformity with really observed different degrees of order of atoms inside a crystal and at its near-surface layers, different reticular densities of particles on different areas of the same face. The constructed model possesses all the attributes of a real crystal: a center, an exterior faceting, symmetries of a certain Fedorov group, finite sizes, and zonality.

\section{Visualization of Lattice Systems}

For computer simulation of crystal structures in the space $R_{E}$ an rCrystal software package has been developed, allowing to conduct the visualization of sections of crystal structures and to construct models of the electrostatic components of parameters of ions. There is the possibility of inputting various types of crystal structures with the use of a special technique of coding. The software package for modeling point systems uses the $F$-algorithm 
for visualizing constructions and the scheme for the interpretation $I_{E}^{R}$ of geometrical objects on the Clifford surface in the Riemannian space.

Examples of the computations of point systems with the use of the $F$-algorithm have been given for modeling the symmetry axes: $l_{3}$ (Figure 4), $l_{4}$ (Figure 5), and $l_{6}$ (Figure 3).

It is obvious from Figure 3(a) and Figure 3(b) that the character of the distributions of the point systems, on the constructed sections perpendicularly to the axis $l_{6}$, essentially differs in a central region. If the point system in Figure 3(a) has a homogeneous distribution, then for the point system in Figure 3(b) a block structure is

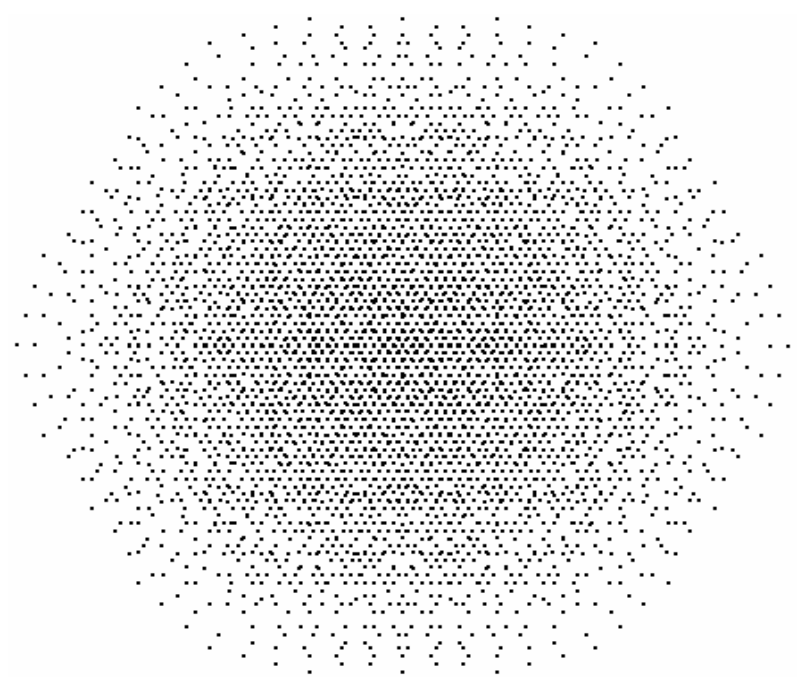

(a)

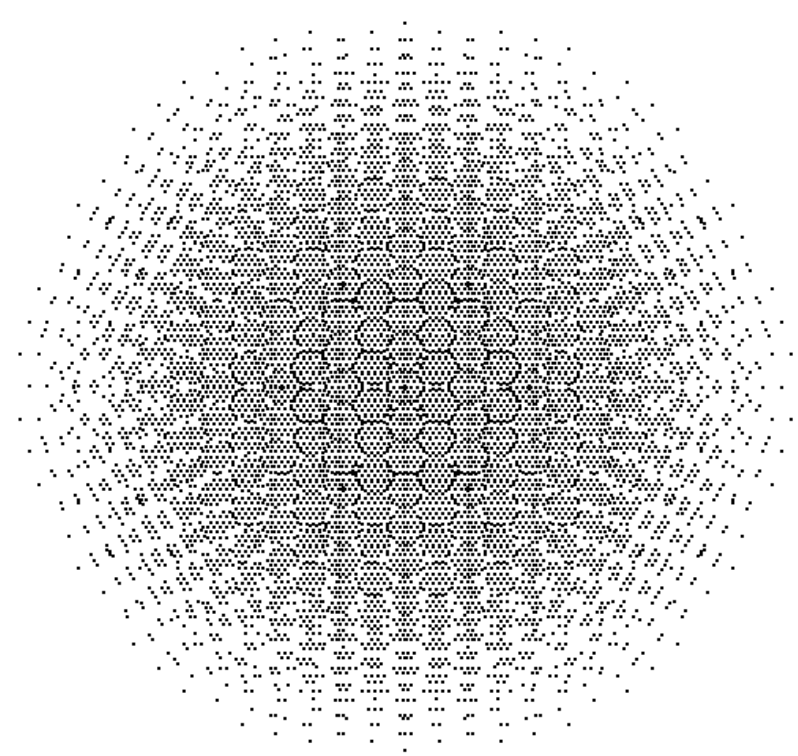

(b)

Figure 3. The homogeneous (a) and block (b) structures (an ideal system) in a section perpendicular to the axes $l_{6}$.

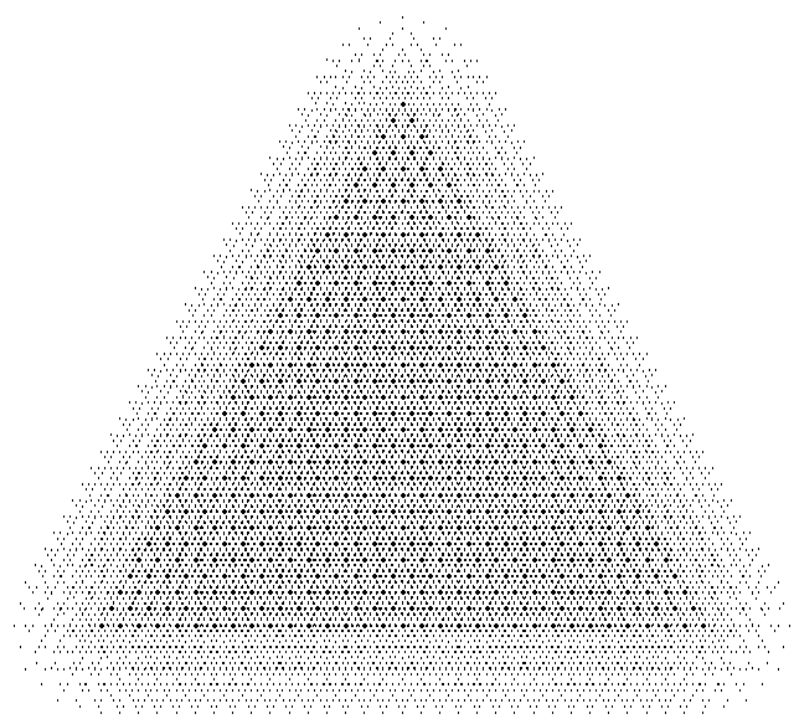

Figure 4. The organization of a lattice system in the section plane, perpendicularly to the axes $l_{3}$, for a model of the trigonal system.

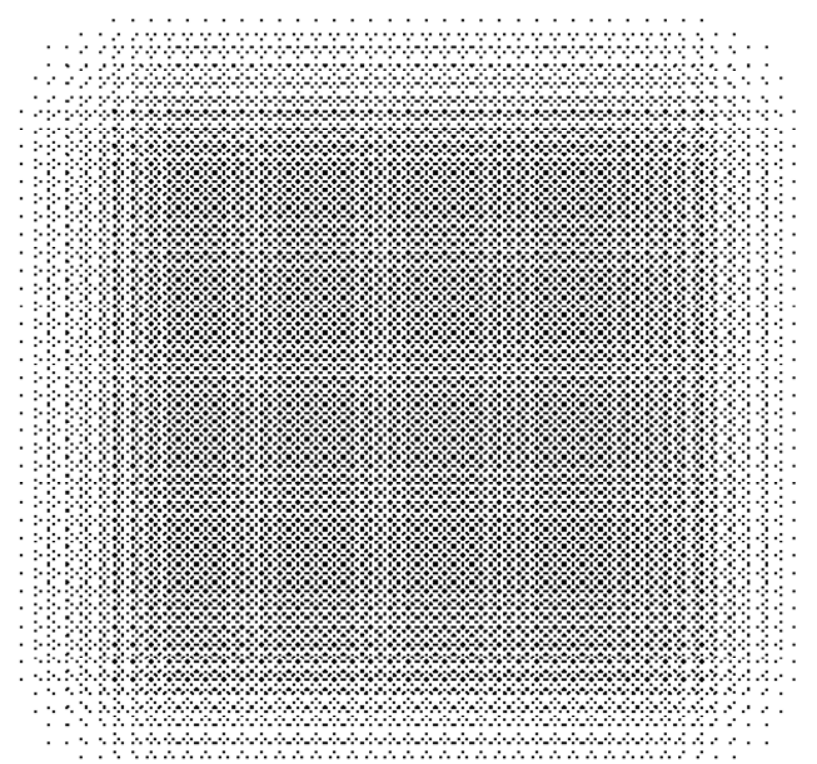

Figure 5. The organization of a lattice system in the section plane, perpendicularly to the axes $l_{4}$, for a model of the cubic system.

clearly seen. All the constructed point systems fill a restricted region in the space and have a centre and a zonal structure.

With the use of the rCrystal software package the complex modeling of the crystal structures $\mathrm{SiO}_{2}$ (Figure 6), $\mathrm{Al}_{2} \mathrm{O}_{3}$ (Figures 7 and 8 ) and a series of its polymorphic modifications $\alpha-, \beta-, \theta$-, and $\gamma-\mathrm{Al}_{2} \mathrm{O}_{3}[17,18]$ has been carried out. A method of modeling mesoatomic ensembles, clusters and crystal structures, permitting to explore the geometrical properties of oxide systems, has been 
developed. On the basis of the model computations a minimum structural element $\mathrm{Al}_{4} \mathrm{O}_{6}$ has been selected, parameters of which were computed in the space $R_{E}$. Using the above mentioned approach a model of the crystalline structure $\mathrm{Al}_{2} \mathrm{O}_{3}$ has been computed. The geometrical configuration of a micropolyhedron $\mathrm{Al}_{4} \mathrm{O}_{6}$ and a set of the external physicochemical conditions (a structure of the electromagnetic field, thermodynamic parameters, crystallophysical parameters) define methods of packing of the micropolyhedrons in different motifs in accordance with the principle of closest packing (Figures 7(a) and (b)). The structure of an electrostatic field modeled in the space $R_{E}$, being formed by the structural element $\mathrm{Al}_{4} \mathrm{O}_{6}$, defines possible methods of integrating into a macrostructure in accordance with the most probable joining mechanism.

\section{Computation of Pair Interaction Potentials When Modeling Structures in the Space $R_{E}$}

In the space $R_{E}$ the models of the electrostatic components of parameters of ions in the case of pair interactions with regard to a charge distribution on a surface have been constructed for the following ions presented in Table 1. When computing, a model of the charged spheres with different charge distributions on a surface was used. The suggested approach uses the scheme $I_{E}^{R}$, what allows to study the features and character of the interaction of elements in a modeled crystal structure, and to explore regularities of its formation.

When computing the geometrical parameters of potentials of the interatomic interaction, it was supposed that the interaction occurs under the conditions of the elliptic

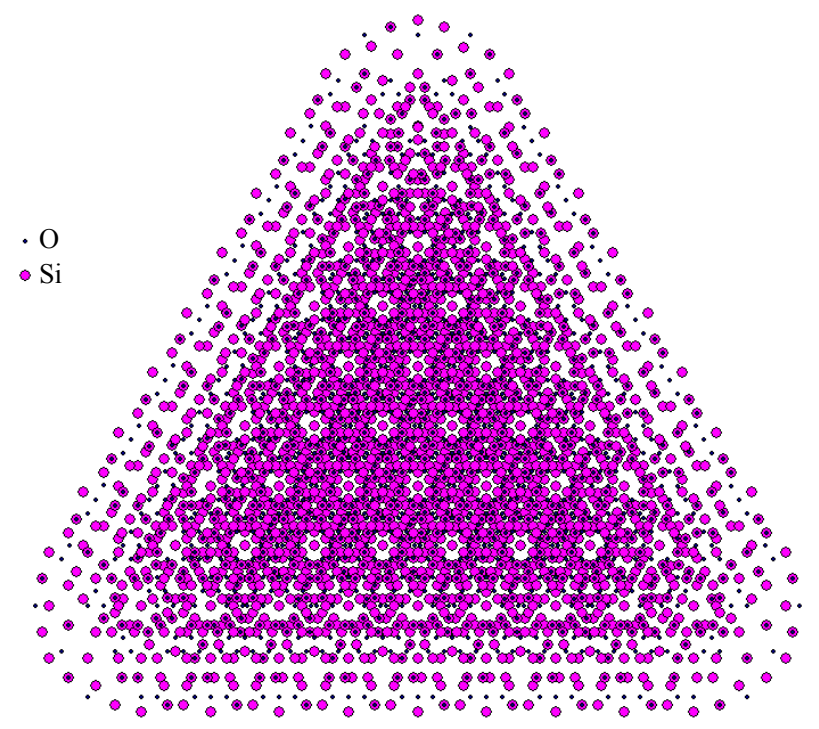

Figure 6. The model of the crystal structure $\mathrm{SiO}_{2}$. The section arranged perpendicularly to the axis $l_{3}$ has been shown. closed Riemannian space $V_{4}$. The structural features of an electrostatic field in the Riemannian space, considered for the case of interacting the unlike charges of ions, impose the special conditions which lead to the formation of coherent states and to the formation of local closed

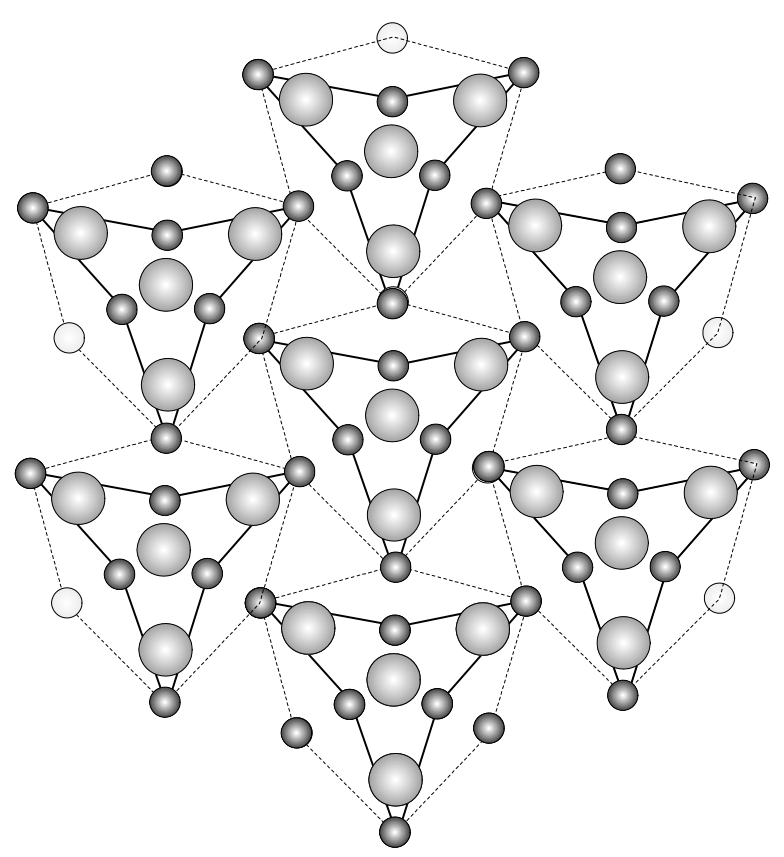

(a)

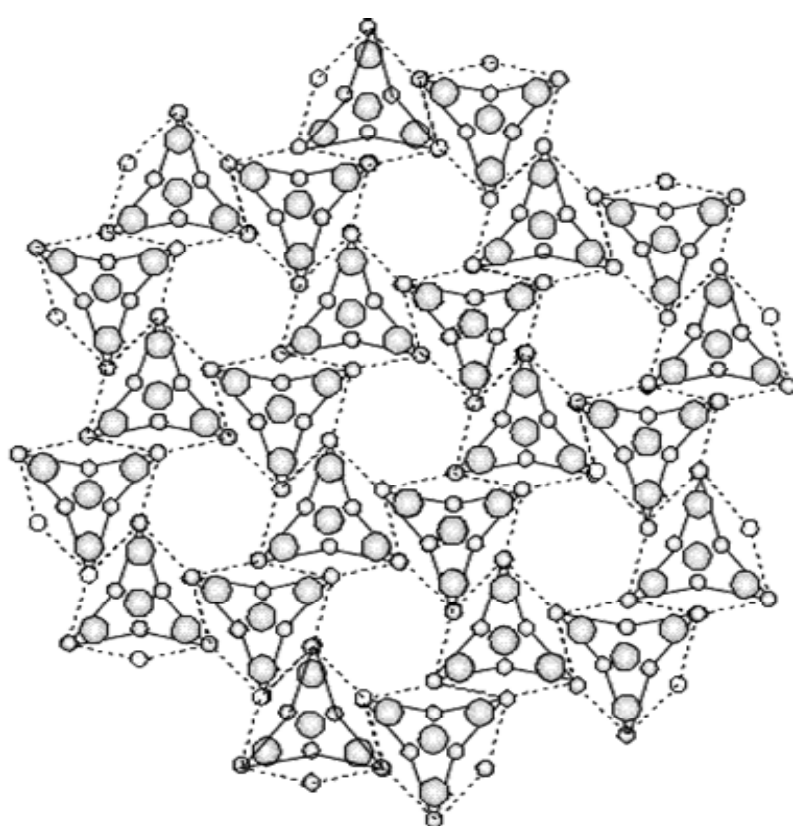

(b)

Figure 7. The motif I1 of the model of the crystal structure $\mathrm{Al}_{2} \mathrm{O}_{3}$, satisfying to the principle of closest packing (a), the corundum motif $I 2$ (b); the models has been presented in a projection on the plane [0001]. 


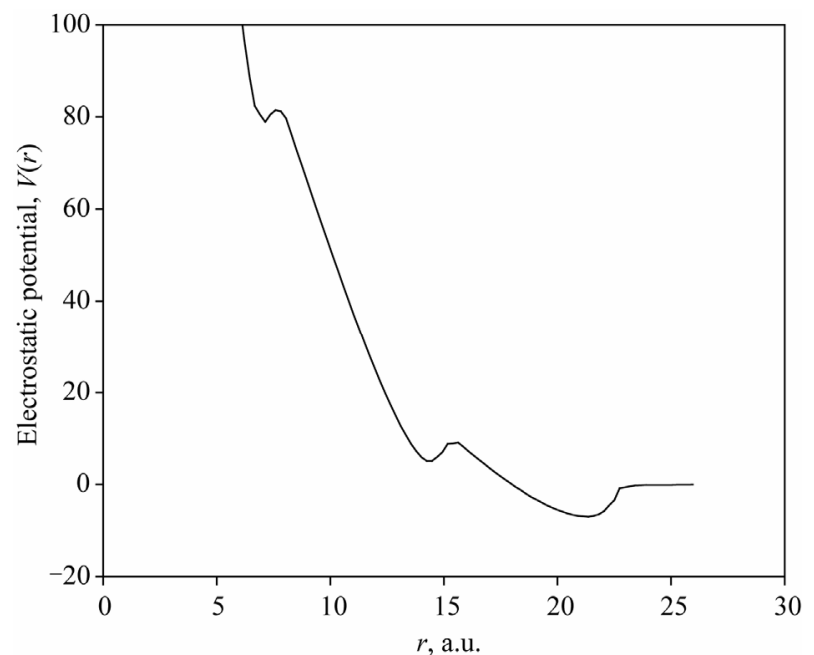

(a)

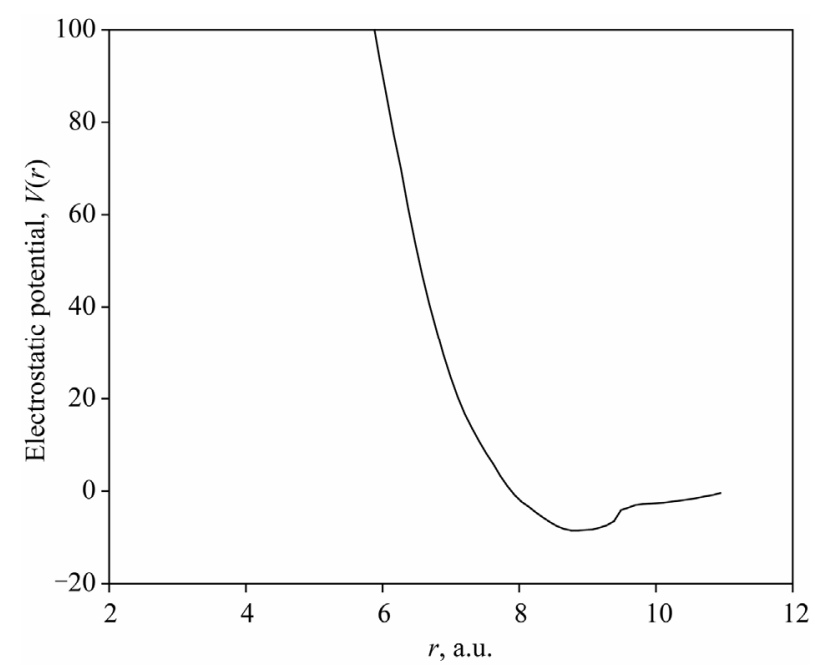

(b)

Figure 8. The model curves of the radial section of the electrostatic potential of the ions: $\mathrm{H}^{+}$(a) and $\mathrm{O}^{2-}(\mathrm{b})$, built in the space $\boldsymbol{R}_{E}$.

Table 1. The computation of radiuses of electrostatic fields of the ions in the space $R_{E}$.

\begin{tabular}{cccc}
\hline ion & $\begin{array}{c}\text { atomic radius } \\
\text { (a.u.) }\end{array}$ & $\begin{array}{c}\text { ionic radius } \\
\text { (a.u.) }\end{array}$ & $\begin{array}{c}\text { radius of } \\
\text { electrostatic field } \\
\text { (a.u.) }\end{array}$ \\
\hline $\mathrm{H}^{+}$ & 0.25 & 1.36 & 23.19 \\
$\mathrm{O}^{2-}$ & 0.66 & 1.36 & 11.04 \\
$\mathrm{Fe}^{2+}$ & 1.18 & 0.78 & 6.26 \\
$\mathrm{Fe}^{3+}$ & 1.18 & 0.65 & 5.79 \\
$\mathrm{Mg}^{2+}$ & 1.45 & 0.72 & 6.87 \\
$\mathrm{Al}^{3+}$ & 1.26 & 0.53 & 5.70 \\
\hline
\end{tabular}

regions of the attraction and repulsion, what speaks of the reasonability of the applied approach $[4,15]$.

In the last column of Table 1 the computed values of radiuses of the electrostatic fields in the space $R_{E}$ have been given. The atomic and ionic radiuses used when modeling were taken from N. Belov's work [19].

When interpreting the total electrostatic field in models of ionic crystals, it becomes possible to take into account the multiparticle and long-range parts of the interactions that occur under the conditions of elliptic Riemannian geometry, and also there appears the possibility for investigating their role in the stabilization of probable polymorphic structures.

The energy of the atomic bunching under the conditions of a given atomic volume in ionic crystals is characterized by the interatomic interaction potential $V(r)$. The suggested model potential becomes strongly repulsive at a distance $R$, less than some critical value $2 R_{0}$ (Figure 8). Let us note that the potential $V(r)$ directly influences on the distance between adjacent ions in a crystal. It can be expected that the distance between the nearest neighbors will be near $R_{\min }$ (a minimum point).

The parameters ${ }^{1}$ of potential curves for a series of the systems under consideration, computed by means of the suggested approach, have been given in Table 2 .

According to the principles of modeling, stated in item 1.3 , the computation of the parameters of components of the electrostatic fields of ions for different elements (Figures 9-11) having participated in the formation of the crystalline sublattices of crystals have been carried out. In the space $R_{E}$ a model of the electrostatic field of a charge is characterized by: a restricted size, the closure, and the continuity. The structure of the model electrostatic field has a distinct zonal character. The shape of a distribution of the energy bands, a group of symmetries and the energy parameters determine a type of the configuration of ions participating in the organization of sublattices.

\section{Conclusions}

The possibilities for constructing a model of a real crystal on other principles immediately connected with the involvement of a non-Euclidean method of describing have been considered. The elliptic closed space of Riemannian geometry $V_{4}$ with the constant Gaussian curvature $K=1$, that coincides in sufficiently small regions with a Euclidean space, was chosen as a modeling space. For visualizing the model constructions the interpretation of geometrical objects on a Clifford surface $\left(S_{\mathrm{K}}\right)$ in Riemannian

\footnotetext{
${ }^{1}$ The Notes: $R_{0}$ is the radius of the hard component of the electrostatic field, where the repulsive forces have a dominating character; $R_{\min }$ is the coordinate of the minimum of the potential $V(r) ; \varepsilon$ is the value of the potential well $V(r)$.
} 
Table 2. The parameters of potential curves of the interatomic interaction in the space $R_{E}$.

\begin{tabular}{lcccc}
\hline bond type & $\begin{array}{c}\mathbf{R}_{\mathbf{0}}, \\
\text { (a.u.) }\end{array}$ & $\begin{array}{c}\mathbf{R}_{\text {min, }} \\
\text { (a.u.) }\end{array}$ & $\begin{array}{c}\text { depth } \\
(\boldsymbol{\varepsilon})\end{array}$ & $\begin{array}{c}\text { radius of } \\
\text { electrostatic } \\
\text { field (a.u.) }\end{array}$ \\
\hline$\left[\mathrm{H}^{+}\right]-\mathrm{O}^{2-}$ & 17.96 & 20.81 & -6.14 & 23.19 \\
{$\left[\mathrm{O}^{2-}\right]-\mathrm{Fe}^{2+}$} & 7.88 & 8.76 & -8.51 & 11.04 \\
{$\left[\mathrm{O}^{2-}\right]-\mathrm{Fe}^{3+}$} & 7.88 & 8.92 & -8.81 & 11.04 \\
{$\left[\mathrm{O}^{2-}\right]-\mathrm{Al}^{3+}$} & 7.98 & 8.97 & -8.90 & 11.04 \\
{$\left[\mathrm{Fe}^{2+}\right]-\mathrm{O}^{2-}$} & 5.07 & 5.68 & -5.81 & 6.26 \\
{$\left[\mathrm{Fe}^{3+}\right]-\mathrm{O}^{2-}$} & 4.71 & 5.51 & -16.22 & 5.79 \\
{$\left[\mathrm{Mg}^{2+}\right]-\mathrm{O}^{2-}$} & 5.60 & 6.24 & -6.32 & 6.87 \\
{$\left[\mathrm{Al}^{3+}\right]-\mathrm{O}^{2-}$} & 4.66 & 5.44 & -17.41 & 5.70 \\
\hline
\end{tabular}

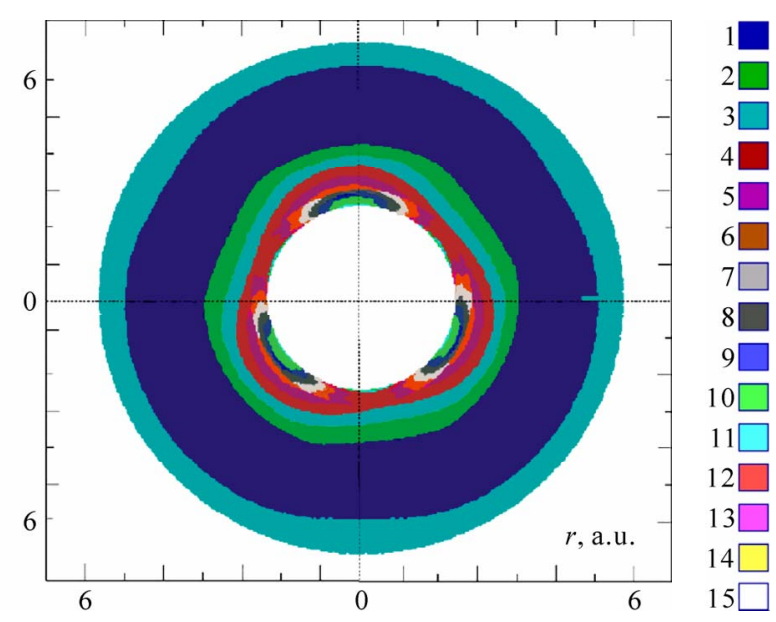

Figure 9. The equatorial section of the model of the electrostatic potential of the ion $\mathrm{Al}^{3+}$. The computation has been carried out in the space $R_{E}$.

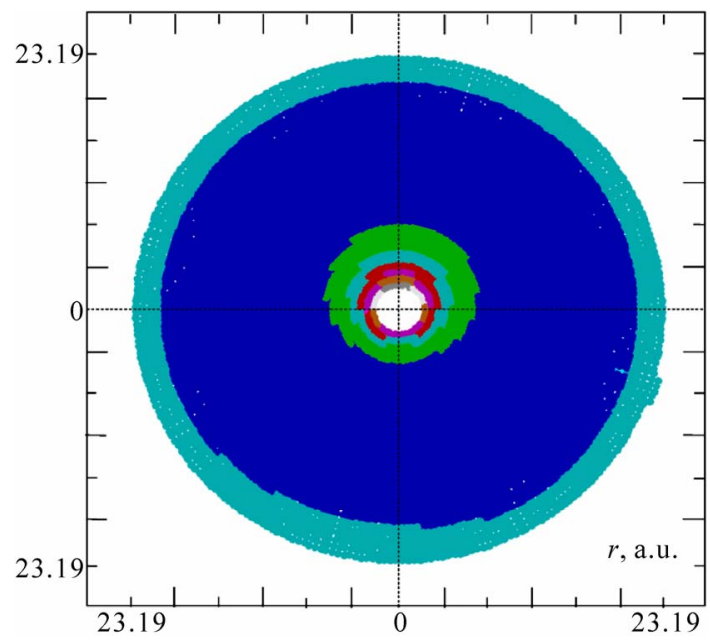

Figure 10. The equipotential picture of the electrostatic field of the ion $\mathrm{H}^{+}$relatively the ion $\mathrm{O}^{2-}$. The frontal section.

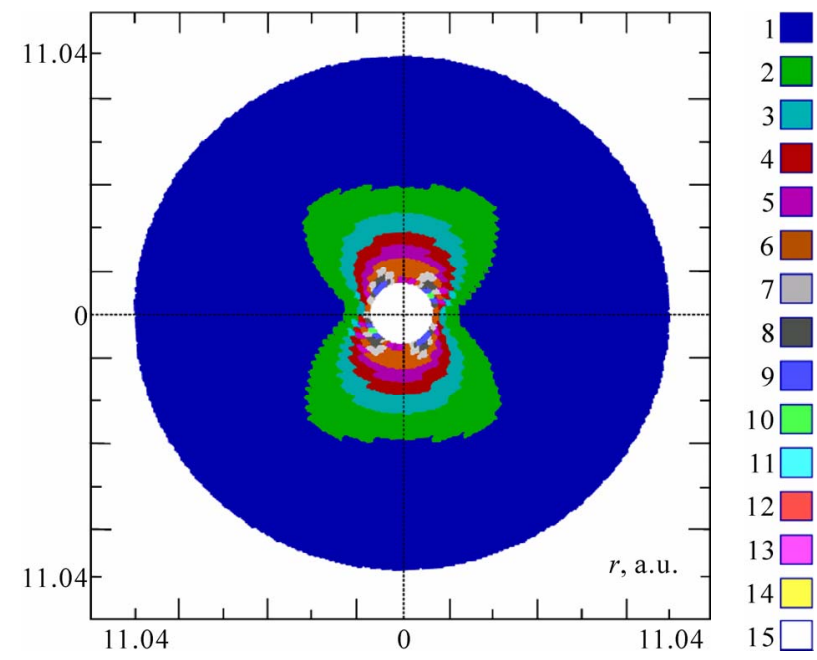

Figure 11. The equipotential picture of the electrostatic field of the ion $\mathrm{O}^{2-}$ relatively the ion $\mathrm{H}^{+}$. The frontal section.

geometry with the help of a 2D torus in a Euclidean space $E_{3}$ is used. The process of the organization of a crystal structure in the space of interpretation $R_{E}$ is presented as a multistage process consisting in sequential introducing the point systems $R=\left\{H_{T}\left(T_{2}\right) K\right\}$ into consideration, each point of which is fixed on a torus in accordance with a given Fedorov group, beginning from a zero point and up to the faceting of a crystal, each of newly fixed point behaving like a new center of the organization of the lattice. Properties of the internal space of a real crystal, such as zonality, boundedness in sizes (finiteness), sectoriality and the occurrence of a center, are naturally deduced from properties of the modeling space. The main characteristic of improvements in the suggested model constructions is that the local changes of structural features, as well as curved regions (defects), can be more completely taken into account.

The developed $F$-algorithm for constructing crystalline lattice systems ensures a computation of 2D sections of models of point systems, arranged perpendicularly to the symmetry axes $l_{3}, l_{4}$, and $l_{6}$. As an example of the realization of the developed principles of modeling, the computations of components of the electrostatic fields of the ions $\mathrm{Al}^{3+}, \mathrm{O}^{2-}, \mathrm{Fe}^{2+}$, and $\mathrm{Mg}^{2+}$, having participated in the formation of the crystalline sublattices of $\alpha-, \beta-, \theta$, and $\gamma-\mathrm{Al}_{2} \mathrm{O}_{3}$, have been presented. The linear parameters of crystal lattices, obtained from the model computations, are connected with the character of their constituent atoms and can be used to determine a type of chemical bonding between them.

The suggested approach can be used for computing the geometrical parameters of the cluster organization of nanostructures of oxides and many other nonequilibrium materials, as well as for the development of practical 
applications connected with improving the structural characteristics of crystalline materials.

\section{REFERENCES}

[1] R. V. Galiulin, "The Geometrical Theory of Crystallization," Crystallography Reports, Vol. 43, No. 2, 1998, pp. 366-347.

[2] S. van Aert, K. J. Batenburg, M. D. Rossell, R. Erni and G. van Tendeloo, "Three-Dimensional Atomic Imaging of Crystalline Nanoparticles," Nature, Vol. 470, 2011, pp. 374-377. doi:10.1038/nature09741

[3] A. Authier, "International Tables for Crystallography: Vol. D, Physical Properties of Crystals," Chapter 1.2, Kluwer Academic Publishers, Dordrecht, 2003.

[4] S. V. Rudnev, "Application of Elliptic Riemannian Geometry to Problems Crystallography," Computers and Mathematics with Applications, Vol. 16, No. 5-8, 1988, pp. 597-616. doi:10.1016/0898-1221(88)90249-0

[5] B. S. Semukhin, A. N. Sergeev and S. V. Rudnev, "Non-Euclidean Interpretation of the Structures of Crystalline Materials," Crystallography Reports, Vol. 4, No. 5, 1999, pp. 738-741.

[6] S. V. Rudnev and V. A Ermolaev, "On Application of Elliptical Riemannian Geometry to Study of Crystalline Structure," Proceedings of Geometry, Vol. 25, 1985, pp. 113-121.

[7] B. S. Semukhin, S. V. Rudnev and R. V. Galiulin, "Application of Riemann Geometry to Structures of Nanoand Macrocrystals," Crystallography Reports, Vol. 53, No. 4, 2008, pp. 541-544. doi:10.1134/S1063774508040019

[8] S. V. Rudnev, A. N. Sergeev, V. G. Bamburov and G. P. Shvykin, "Geometrical Modeling of Superplastic Constructional Ceramics Structure," Doklady Chemistry, Vol. 341, No. 4, 1995, pp. 589-501.

[9] S. A. Bogomolov, "An Introduction to Non-Euclidian Geometry,” GTTI, Moscow-Leningrad, 1934.

[10] W. K. Clifford, "Preliminary Sketch of Biquaternions," Proceedings of the London Mathematical Society, Vol. S1-S4, No. 1, 1871, pp. 381-395. doi:10.1112/plms/s1-4.1.381
[11] H. A. R. Aliabad and S. R. Ghorbani, "Structural and Spin Polarization Effects of $\mathrm{Cr}$, Fe and Ti Elements on Electronical Properties of $\alpha-\mathrm{Al}_{2} \mathrm{O}_{3}$ by First Principle Calculations," Journal of Modern Physics, Vol. 2, No. 3, 2011, pp. 158-161. doi:10.4236/jmp.2011.23024

[12] J. Z. Sun, T. Stirner and A. Matthews, "Structure and Electronic Properties Calculation of Ultrathin $\alpha-\mathrm{Al}_{2} \mathrm{O}_{3}$ Films on (0001) $\alpha-\mathrm{Cr}_{2} \mathrm{O}_{3}$ Templates," Surface Science, Vol. 601, No. 21, 2007, pp. 5050-5056. doi:10.1016/j.susc.2007.09.004

[13] M. M. Zhong, X. Y. Kuang, H. Q. Wang; H. F. Li, Y. R. Zhao, "Density Functional Study of the Structural and Electronic Properties of Tetra-Aluminum Oxide $\mathrm{Al}_{4} \mathrm{O}_{\mathrm{n}}^{\lambda}$ (3 $\leq n \leq 8, \lambda=0,-1)$ Clusters," Molecular Physics: An International Journal at the Interface between Chemistry and Physics, Vol. 109, No. 4, 2011, pp. 603-612. doi: 10.1080/00268976.2010.542777

[14] R. Ahuja, J. M. R. Ahuja, J. M. Osorio-Guillen, J. Souza de Almeida, B. Holm, W. Y. Ching and B. Johansson, "Electronic and Optical Properties of $\gamma-\mathrm{Al}_{2} \mathrm{O}_{3}$ from $a b$ Initio Theory, "Journal of Physics: Condensed Matter, Vol. 15, No. 16, 2004, pp. 2891-2898. doi: 10.1088/0953-8984/16/16/013

[15] S. V. Rudnev and A. N. Sergeev, "ICS Simulation of Crystal Mineral Grouth and Deformation," TGU Tomsk State University, Tomsk, 1994.

[16] B. A. Dubrovin, A. T. Fomenko and S. P. Novikov, "Modern Geometry-Methods and Applications: Part I: The Geometry of Surfaces, Transformation Groups, and Fields," Springer-Verlag, New York, 1991.

[17] A. P. Klishin, A. N. Zakutaev, S. V. Rudnev, V. A. Ermolaev and T. A. Habas, "Modeling Process of Structural Transformations in $\mathrm{Al}_{2} \mathrm{O}_{3}$ at Magnetothermal Treatment," Constructions from Composite Materials, Vol. 1, 2008, pp. 12-17.

[18] A. P. Klishin, S. V. Rudnev and V. I. Vereshagin, "New Technological Approaches of Reception Alumina Materials with Usage of Magnetothermal Treatment," Refractories and Technical Ceramics, No. 6, 2009, pp. 30-34.

[19] N. V. Belov, "Structural Crystallography," USSR Academy of Sciences, Moscow, 1952. 